\title{
Vasogenic edema due to tight junction disruption by matrix metalloproteinases in cerebral ischemia
}

\author{
Gary A. Rosenberg, M.D., ${ }^{1,2,3}$ ANd Yi YAng, M.D., Ph.D. ${ }^{1}$ \\ Departments of ${ }^{1}$ Neurology, ${ }^{2}$ Neurosciences, and ${ }^{3}$ Cell Biology and Physiology, University of \\ New Mexico Health Sciences Center, Albuquerque, New Mexico
}

\begin{abstract}
$\checkmark$ Cerebral ischemia causes cell swelling and breakdown of the blood-brain barrier (BBB). Cytotoxic edema results from energy failure, and vasogenic edema occurs when the blood vessels are damaged. Proteases and free radicals are the end result of a molecular injury cascade. Matrix metalloproteinases (MMPs) are a gene family of extracellular matrix-degrading enzymes that disrupt the BBB. Tight junction proteins (TJPs), occludin and claudin-5, which form the endothelial barrier, are vulnerable to attack by MMPs. Basal lamina proteins, such as fibronectin, laminin, and heparan sulfate, are also degraded by MMPs. Reperfusion injury leads to a biphasic opening of the BBB, with the early opening occurring several hours after the onset of reperfusion due to activation of the constitutive enzyme gelatinase A (MMP2). This initial opening is transient and followed 24 to 48 hours later by more intense damage to the blood vessel, which is associated with the expression and activation of gelatinase B (MMP-9) and stromelysin-1 (MMP-3). Synthetic MMP inhibitors restore the early integrity of the BBB but are ineffective in the later opening. Because these inhibitors block MMPs involved in angiogenesis and neurogenesis, they also slow recovery. The challenge is to identify agents that will protect the BBB, blocking vasogenic edema without interfering with recovery.
\end{abstract}

KEY WORDS - blood-brain barrier • edema • ischemia • matrix metalloproteinase • neuroinflammation $\bullet$ tight junction protein

$\mathrm{V}$ ASOGENIC EDEMA, which occurs due to breakdown of the BBB in cerebral ischemia, is aggravated by reperfusion. ${ }^{4,13,16,37}$ It has been recognized that hemorrhagic transformation after ischemic infarction occurs more commonly with embolism than with thrombosis, presumably because of clot resolution through reperfusion. ${ }^{9,15}$ Increased interest in the mechanisms of BBB damage and vasogenic edema was generated by the introduction of clot lysis with rt-PA, which increased the risk of symptomatic intracerebral hemorrhage., 2,36 The safety of rt-PA treatment was improved with agents that prevent the extravasation of the enzyme into the brain across a damaged BBB. Disruption of the BBB is a complex process that involves multiple layers of cell processes and proteins. Tight junction proteins between endothelial cells form the initial interface. Surrounding the endothelial cells is a basal lamina composed of extracellular matrix proteins. Embedded in the basal lamina is a macrophage-

Abbreviations used in this paper: $\mathrm{BBB}=$ blood-brain barrier; MCAO = middle cerebral artery occlusion; LPS = lipopolysaccharide $; \mathrm{MMP}=$ matrix metalloproteinase $\mathrm{MR}=$ magnetic resonance; MT1-MMP = membrane type $1 \mathrm{MMP}$; rt-PA = recombinant tissue plasminogen activator; TIMP $=$ tissue inhibitor of metalloproteinase; TJP = tight junction protein. like pericyte that has smooth muscle properties. Finally, the foot processes of the astrocytes form the outer layer. This series of layers is referred to as the "BBB," or more recently as the "neurovascular unit."

Data from earlier studies have shown that enzymes are capable of proteolytically degrading the extracellular matrix around blood vessels; these enzymes include plasmin, chondroitinase, and collagenase. ${ }^{26}$ We have shown that the injection of bacterial collagenase into the caudate region in the rat leads to extensive bleeding. ${ }^{31}$ Pursuing that observation further, we showed that the intracerebral injection of purified mammalian Type IV collagenase, which had been isolated from metastatic melanoma cells, opened the BBB and caused localized bleeding around the vessel. The hemorrhages resembled "ball hemorrhages" similar to those seen in hemorrhagic transformation, suggesting that reperfusion injury may be due to a proteolytic attack. ${ }^{30}$ This finding led to a series of studies focused on isolating the Type IV collagenases in injured brain, culminating in the revelation that ischemic injury caused an increase in two Type IV collagenases, gelatinase A (MMP-2) and gelatinase B (MMP-9), along with plasminogen activators. ${ }^{32}$

Type IV collagenases are members of a larger MMP gene family of proteolytic enzymes that play a critical role in development as well as in pathological processes such 

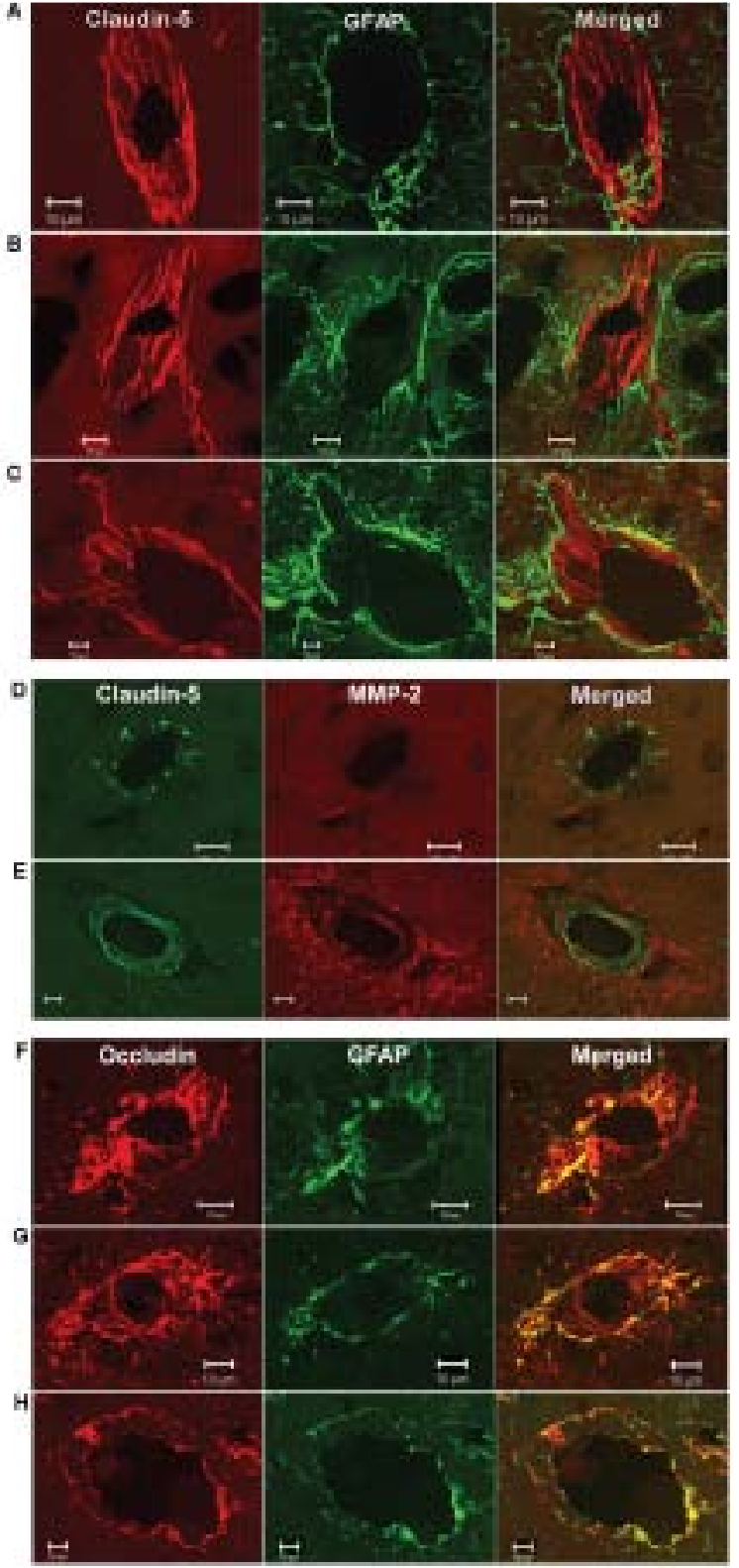

FIG. 1. Dual-labeled fluorescent confocal micrographs of the piriform cortex from spontaneously hypertensive rats that had undergone 90 minutes of MCAO and 3 hours of reperfusion, showing claudin5 and occludin immunoreactivity of TJPs. A sham brain (A) and a nonischemic side of the brain (B) reveal that the claudin-5 (Cy3) in blood vessels is separated from the astrocytes (glial fibrillary acidic protein [GFAP]) surrounding them. The merged images make the separation of the claudin-5 and astrocytes very evident. In an ischemic hemisphere (C) there is fragmentation and degeneration of claudin-5 immunoreactivity. Colocalization of claudin-5 and GFAP was seen in the ischemic hemisphere. A nonischemic side (D) shows that minimal MMP-2 immunoreactivity was detected in blood vessels where claudin-5 presented straight rows of staining, which are similar to those seen in the nonischemic cortex featured in Fig. 5B. The MMP-2 immunoreactivity surrounding blood vessels colocalized with claudin-5 in the ischemic hemisphere (E) after 3 hours of reperfusion. Occludin immunoreactivity in the sham brain $(\mathrm{F})$ and nonischemic piriform cortex $(\mathrm{G})$. The nonischemic side shows that the occludin (Cy3) and the astrocytes (GFAP) were colocalized around blood vessels. Occludin immunostaining in ischemic piriform cortex $(\mathrm{H})$ was characterized by degeneration and reduced expression compared with that in nonischemic piriform cortex. Bars $=10$ $\mu \mathrm{M}$. (from Yang et al., 2006; reprinted with permission from Elsevier). as metastasis of cancer, arthritic destruction of collagen in joints, rupture of atherosclerotic plaques in the heart and carotid artery, acute inflammation in multiple sclerosis, vasogenic edema in stroke, brain infections, and brain trauma. ${ }^{39}$ In stroke, the MMPs as well as cytokines and free radicals are induced as part of an inflammatory response related to hypoxia. ${ }^{28}$ The action of MMPs is regulated by four endogenous TIMPs. ${ }^{7}$ In addition to their destructive role in stroke, MMPs and TIMPs are important in the recovery phase when they facilitate angiogenesis and neurogenesis. Because of the wide range of diseases that involve pathological changes in the MMPs, there is great interest in the development of synthetic inhibitors of MMPs that could be used in therapy. These endeavors have met with mixed success because of the complex roles that MMPs play not only in injury but also in normal healing processes.

\section{Proteolysis of TJPs by MMPs}

Interfaces between the systemic circulation and brain tissues preserve the internal milieu of the brain. ${ }^{1}$ The BBB tightly regulates the brain fluid environment by controlling the transport of substances into and out of the brain. The junction proteins, which form the tight junctions between the endothelial cells, assemble in the clefts of the cerebral blood vessels to restrict transport across the BBB. Several TJPs have been isolated and cloned. Within the cerebral endothelial cells are zona occludin molecules anchored to the actin cytoskeleton. Claudins form the tight junctions between the endothelial cells. Occludin is another of the TJPs within the clefts between the endothelial cells. At the level of the endothelial cells, which are directly in contact with the systemic circulation, the TJPs form a seal that blocks the entry of large proteins and charged 
A

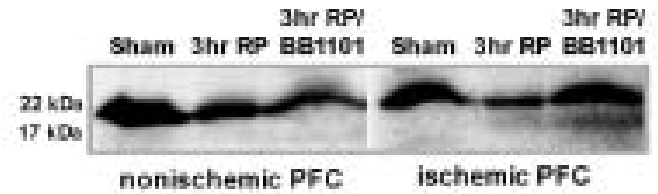

Claudin-5

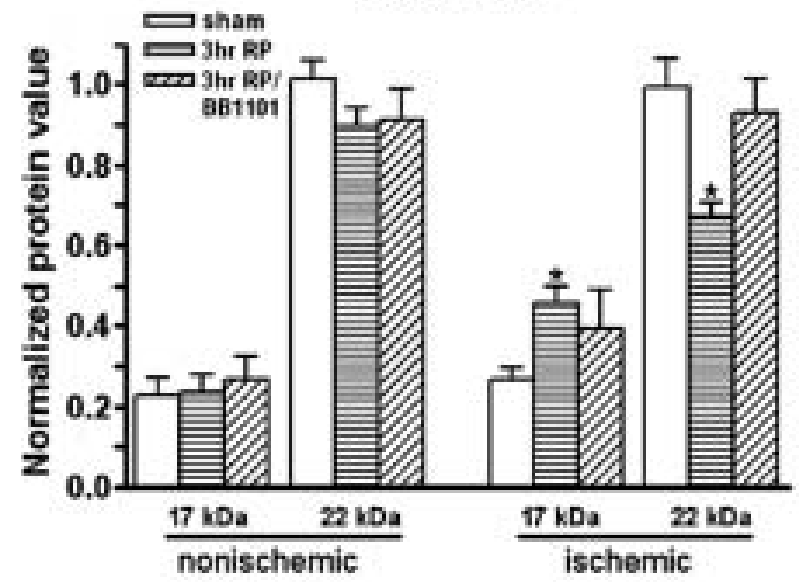

B

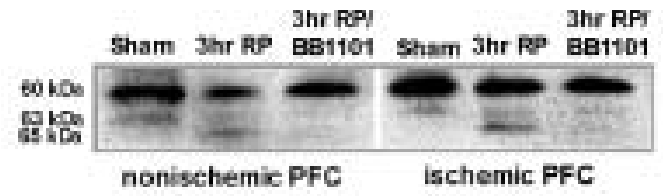

Occludin

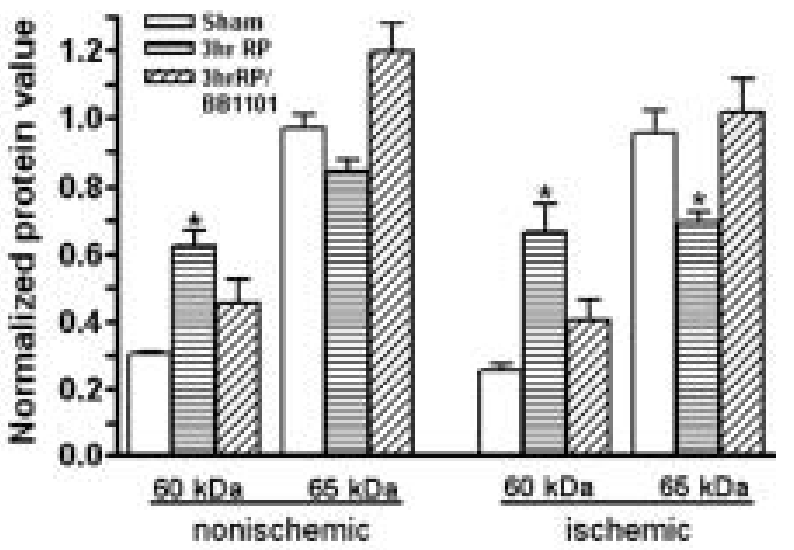

FIG. 2. Western blots and bar graphs showing the degradation of TJPs after a 90-minute MCAO followed by 3 hours of reperfusion. A: There were significant reductions (six rats, $* \mathrm{p}<0.01)$ in claudin-5 $(22-\mathrm{kD}[\mathrm{kDa}]$ band) in the ischemic hemisphere at 3 hours after reperfusion ( $3 \mathrm{hr} \mathrm{RP}$ ). In addition, a new $17-\mathrm{kD}$ band for claudin-5 was found in the ischemic hemisphere 3 hours postreperfusion. The $17-\mathrm{kD}$ protein shows a significant increase $(* \mathrm{p}<0.01)$.

Treatment with the broad-spectrum MMP inhibitor BB-1101 significantly reversed the reduction in claudin-5 (22-kD band; six rats, $p<0.02$ ), indicating that TJPs are degraded by MMPs. B: There was a significant reduction in occludin $\left(65-\mathrm{kD}\right.$ band) in the ischemic hemisphere at 3 hours of reperfusion (six rats, $\left.{ }^{*} \mathrm{p}<0.02\right)$; treatment with BB1101 reversed this reduction (six rats, $\mathrm{p}<0.02$ ). A lower $60-\mathrm{kD}$ band was induced in both nonischemic and ischemic hemispheres ( $\mathrm{p}<0.003$ and $\mathrm{p}<0.02$, respectively). The BB-1101 blocked the appearance of the $60-\mathrm{kD}$ band (six rats, $\mathrm{p}<0.05$ ). $\mathrm{PFC}=$ piriform cortex (from Yang et al., 2006, reprinted with permission from Elsevier).

molecules. ${ }^{10,14}$ The TJPs, including claudins and occludin, are proteolytically degraded by the MMPs. ${ }^{38}$ In a suture stroke model in spontaneously hypertensive rats, a 90minute MCAO with 3 hours of reperfusion resulted in cleavage of TJPs by activated MMP-2. Degradation of the TJPs was blocked by a synthetic broad-spectrum MMP inhibitor.

Gelatinase A, or MMP-2, is a constitutively expressed MMP that is normally present in the brain in a latent form and can be detected in the cerebrospinal fluid. The mechanism of MMP-2 activation involves the formation at the cell surface of a trimolecular complex of pro-MMP-2, MT1-MMP, and TIMP-2. ${ }^{33,34}$ Small amounts of TIMP-2 facilitate activation of MMP-2, whereas larger amounts inhibit activation. The MT1-MMP is activated by the intracellular proprotein convertase furin. ${ }^{6}$ All components necessary to activate MMP-2 are either present or induced within 3 hours after the onset of reperfusion. In the rats exposed to 90 minutes of MCAO, there was transient $\mathrm{BBB}$ opening 3 hours after the initiation of reperfusion. Using confocal microscopy, we have observed fragmentation of the TJPs within 3 hours, which differed from the arrayed linear structures that seemed to be "ziplocked" together (Fig. 1).

Western blotting, which was done to confirm the disruption seen on confocal microscopy, showed cleavage of the two major TJPs: claudin-5 and occludin. This breakdown could be prevented by treatment with a broad-spectrum MMP inhibitor, which adds further support to a role for MMP-2 in TJP degradation (Fig. 2). After its transient early opening, the BBB remained relatively intact until 24 to 48 hours later, when a more disruptive opening occurred. We observed that by 24 hours the TJPs had moved out of the vasculature into the adjacent astrocytes. We were unable to determine whether the TJPs were formed by the astrocytes or taken up by them after being degraded.

The restored integrity of the BBB after its early opening suggests that TJPs are initially maintained within the blood vessel clefts and would remain intact if the later, more damaging stage of injury could be blocked. At later times the inflammatory response leads to the induction of MMP-3 and MMP-9. Matrix metalloproteinases are secreted as latent enzymes that require activation. In particular, MMP-9 is activated in the extracellular space by other proteases and free radicals. Results of in vitro studies have suggested that MMP-3 activates MMP-9, but the mechanisms in vivo are less well understood. ${ }^{21}$

\section{Role of MMP-3 in Neuroinflammation}

We have studied the role of MMP-3 in a model of neuroinflammation involving the intracerebral injection of 


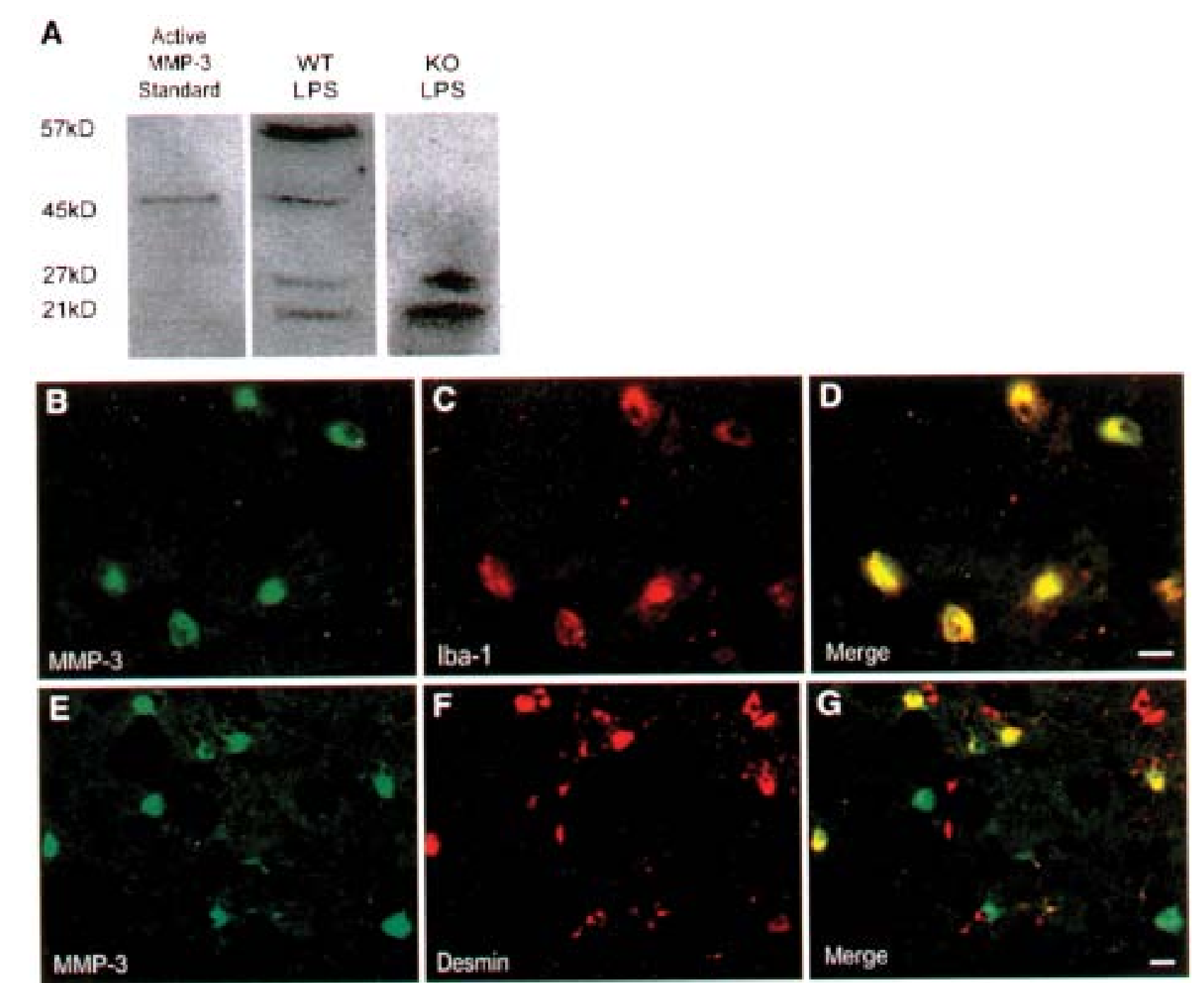

FIG. 3. Western gel blots and photomicrographs revealing the effects of MMP-3 on inflammation.

Lipopolysaccharide was injected into the caudate nucleus, and tissue around the injection site was removed 24 hours later for analysis of protein expression. The LPS induced an MMP-mediated disruption of the BBB. A: Western blotting for MMP-3 showed the proform at $57 \mathrm{kD}$ and the active form at $45 \mathrm{kD}$ along with two lower, unidentified bands. The MMP-3 knockout mouse did not show the 57- or 45-kD bands on staining with MMP-3. The MMP-3 colocalized with Iba-1-immunoreactive microglia/macrophages. B: Note the MMP-3 staining in sham brain. C: An immunostain for microglia/macrophages in mice, Iba-1, was positive. D: Merged image of panels B and C. In pericytes stained for desmin, MMP-3 was seen in LPS-injected wild-type mice. E: Staining with MMP-3. F: Desmin staining. G: Merged composite of panels E and F. KO =knockout; WT = wild type. Bars = $10 \mu \mathrm{m}$ (from Gurney et al., 2006).

LPS. ${ }^{12}$ The region around the injection site becomes inflamed and a small region of BBB damage is induced. We have shown that opening the $\mathrm{BBB}$ in this model involves the induction and activation of the proinflammatory enzymes MMP-3 and MMP-9. Using immunohistochemistry, we found that MMP-3 is colocalized to the pericytes and microglia (Fig. 3). Neutrophils contain formed MMP9 that is released in packets during inflammation. ${ }^{24} \mathrm{We}$ observed the neutrophils around the site of injection. Given that both MMP-9 and MMP-3 were in the vicinity of the blood vessels, there may have been an interaction between them.

We further explored the role of MMP-3 by using mice in which the MMP-3 gene had been knocked out. ${ }^{20}$ Neutrophils around the site of injection were counted with unbiased stereological methods. The MMP-3 knockout mice had fewer neutrophils than the wild type, suggesting that the MMP-3 contributed to opening of the BBB either by directly attacking the basal lamina around the blood vessels or by activating MMP-9, which in turn attacked the extracellular matrix proteins (Fig. 4). We found that MMP-3 affected the TJPs; the MMP-3 knockout mice had smaller losses of claudin-5 and occludin than did the wild type (Fig. 5). Furthermore, we showed that MMP-3 attacked proteins in the basal lamina, such as laminin, given that the MMP-3 knockout mice had larger amounts of 


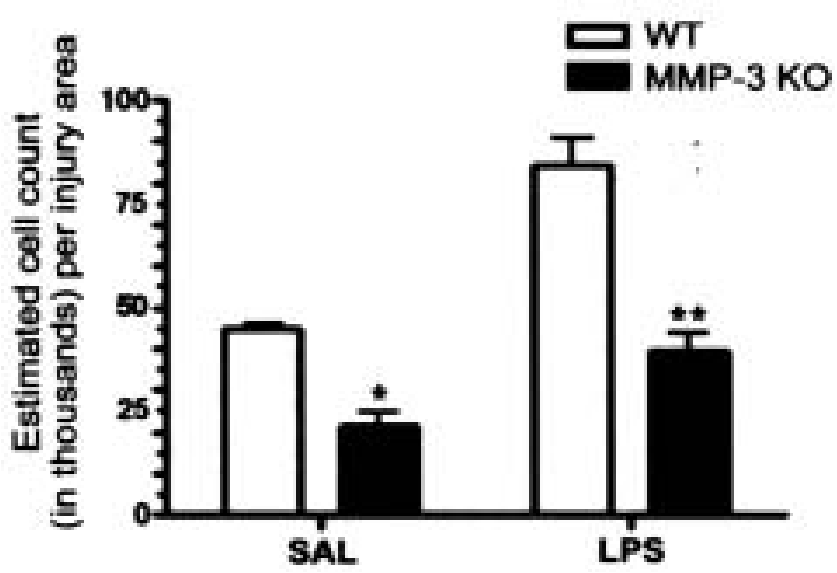

FIG. 4. Bar graph demonstrating results of stereological methods for neutrophil counts 24 hours after LPS or saline (SAL) injection into the caudate nucleus. Counts of myeloperoxidaseimmunoreactive neutrophils were greater in the LPS-injected caudate nucleus in both wild type (WT) and MMP-3 knockout (KO) mice. In the MMP-3 knockout mice, however, there were significantly fewer neutrophils in the caudate nucleus compared with those in the wild type mice, in both saline- and LPS-injected hemispheres. Four mice for each WT and KO group. ${ }^{*} \mathrm{p}<0.01$, $* * \mathrm{p}<0.001$. (From Gurney et al., 2006.)

laminin than did the wild type (Fig. 6). Most likely, the MMP-3 directly attacked the laminin considering that it is a known substrate of the enzyme, although it is possible that other MMPs, such as MMP-9, contributed to the attack on the TJPs. Because they are also formed as latent enzymes, a mechanism for activation is needed.

When the inflammatory MMPs are present, they are free to move within the extracellular space, where they could create greater damage than those attached to the cell surfaces. Although the studies in the MMP-3 knockout mice were done using an inflammation model rather than an ischemic one, the mechanisms of damage to the BBB are probably similar between the two types of injuries and could explain the marked loss of TJPs from the cerebral endothelial cells seen at the time of the second opening of the BBB. The early opening was dominated by the MMP2 bound to the cell surface by the MT1-MMP, which restricted damage to the vicinity of the cell surface in the immediate region. When the more generalized inflammatory response took place at 24 to 48 hours, there was a release of the unbound MMP-9 and MMP-3, and the damage caused by the unconstrained proteases in the extracellular space was more extensive.

\section{Role of MMPs in rt-PA-Induced Intracerebral Bleeding}

Treatment of stroke with rt-PA improves outcome. ${ }^{2}$ Although the incidence of hemorrhagic transformation is similar in treated and untreated patients, there is a greater risk of symptomatic intracerebral hemorrhage, resulting in morbidity and death, in treated patients. ${ }^{8}$ The reason for this phenomenon has been unraveled over the past several years. Normally there is an incidence of hemorrhagic transformation that is estimated to be as high as $60 \%$ in embolic stroke. ${ }^{15}$ Reperfusion in itself has been recognized for more than 50 years as a major cause of bleeding in the brain. ${ }^{9}$ The rt-PA in blood is prevented from entering the brain by the BBB. In the early stages of infarction, there is a transient opening of the BBB. When rt-PA is given by 3 hours after the onset of stroke, as is recommended, the BBB is compromised and rt-PA extravasates into the brain. Once in contact with brain cells, the rt-PA activates the MMPs, causing an attack on the BBB with hemorrhage. ${ }^{19}$ Thus, the beneficial effect of rt-PA requires that it remain within the vessels.

Synthetic inhibitors of MMPs block the opening of the BBB. ${ }^{29}$ Inhibitors have been developed to block the MMPs involved in the metastasis of cancer, and most of the clinical trials have been focused on cancer treatment. ${ }^{5,22}$ The most effective MMP inhibitors currently used are hydroxymate-based compounds. ${ }^{11}$ Data in clinical trials have shown some benefit for their use in the treatment of advanced cancer, but side effects related to joint pain due to the loss of extracellular matrix remodeling have forced the closure of these trials. Given the role of the MMPs in the early opening of the BBB and in hemorrhage, it was reasoned that these agents would be useful in rt-PA treatment. An MMP inhibitor was tested in the multiple emboli model of stroke and was shown to reduce the number of hemorrhages. ${ }^{17}$

Personnel at our laboratory have studied the effect of a broad-spectrum MMP inhibitor, BB-94, administered intraperitoneally in rats. ${ }^{25}$ Animals with MCAO for various times underwent reperfusion. After 1.5 hours of occlusion and 16.5 hours of reperfusion, only 1 of 12 animals died, even when given $10 \mathrm{mg} / \mathrm{kg}$ of rt-PA, which is the dose used in rats (Fig. 7A). After 6 hours of occlusion and 12 hours of reperfusion, however, death occurred in a significantly increased number of animals, that is, one of six animals without treatment and five of six with rt-PA treatment. When BB-94 was given shortly before treatment with rt-PA in animals that had undergone 6 hours of occlusion and 12 hours of reperfusion, the death rate was decreased significantly. The effect of reperfusion on the $\mathrm{BBB}$ was studied with ${ }^{14} \mathrm{C}$-sucrose. After 6 hours of occlusion and 1 hour of reperfusion, the sucrose space (the ratio of sucrose in the brain to that in the blood) was greater than 7\%. Treatment with BB-94 significantly reduced the opening (Fig. 7B). This result indicates that the protection provided by the MMP inhibitor was most likely attributable to the prevention of opening the BBB and the inability of the rt-PA to enter the brain.

\section{Matrix Metalloproteinase Inhibitors for Treatment of Vasogenic Brain Edema}

The toxicity of rt-PA occurs when there is extravasation of rt-PA from the blood into the brain, where it activates glutamate receptors in neurons and increases the activity of the MMPs..$^{23,35}$ The challenge is to identify the optimal MMP inhibitor and the appropriate time window for its use. Authors of several studies have identified MMP inhibitors as possible treatments for vasogenic edema. In an earlier study, we found that another broad-spectrum MMP inhibitor, BB-1101, was also effective. ${ }^{29}$ Both of these 
A Claudin-5

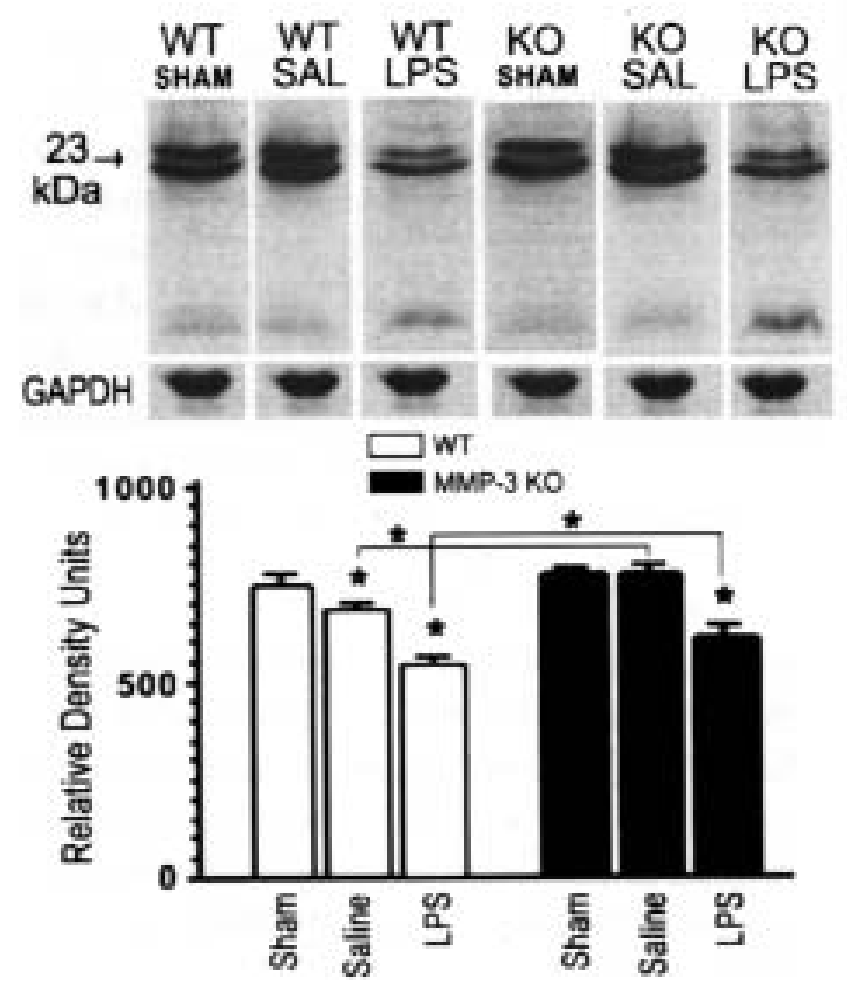

B

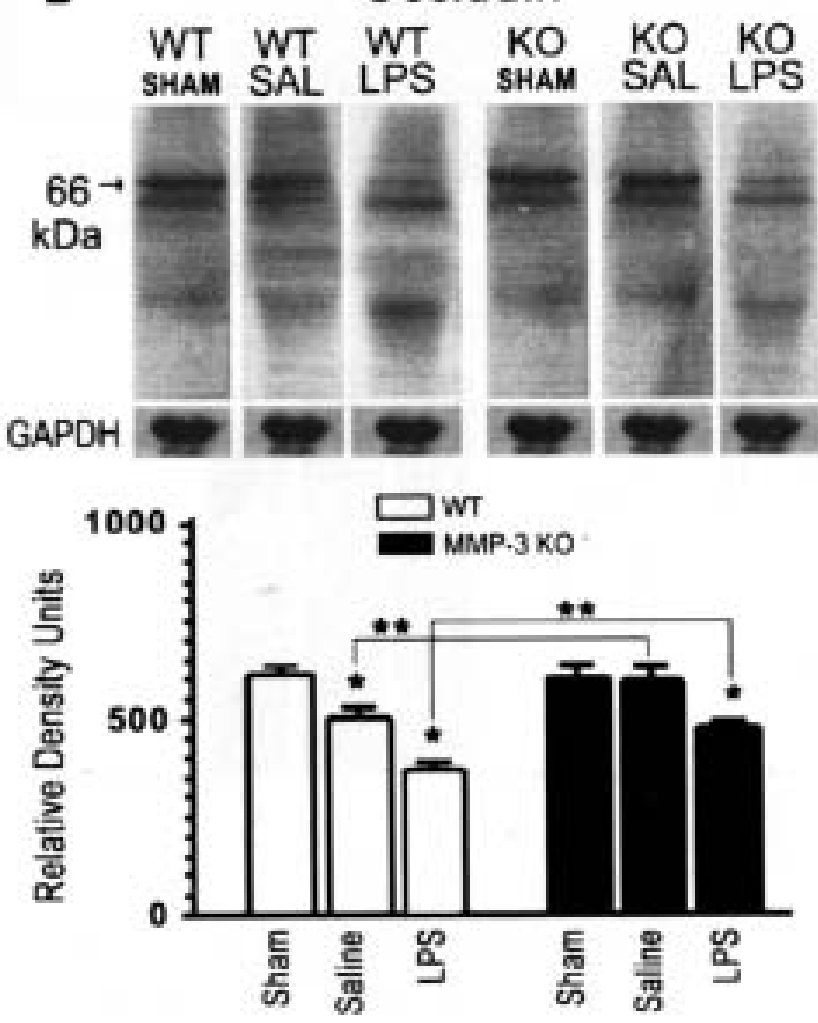

FIG. 5. Western gel blots and bar graphs showing the effect of LPS injection on TJPs in MMP-3 knockout and wildtype mice. Representative bands from one mouse are shown in the upper portion of each figure. The major forms of 23 $\mathrm{kD}$ claudin-5 and $66 \mathrm{kD}$ occludin were measured using densitometry. A: Twenty-four hours after LPS or saline injection, it became apparent that the LPS injection resulted in a significant decrease in claudin-5 levels in both wild-type (WT) and MMP-3 knockout (KO) mice (three animals for each group, $* \mathrm{p}<0.05$ ). B: Measurements of occludin also decreased after LPS injection. However, claudin-5 and occludin levels decreased to a lesser extent in the MMP-3 knockout than in wild-type mice $(* \mathrm{p}<0.05$ and $* * \mathrm{p}<0.001)$. The lower bands on each Western blot are probably degradation products. GAPDH = glyceraldehyde-3-phosphate-dehydrogenase. (From Gurney et al., 2006.)

agents are very poorly soluble, making it unlikely that they will be used in clinical trials.

Confounding the use of the MMP inhibitors is the fact that MMPs play an important role in the recovery phase and possibly in the normal turnover of extracellular matrix. In a recent study, the authors showed that MMPs are critical for recovery because they are involved in both angiogenesis and neurogenesis; MMP inhibitors slowed recovery after cerebral infarction. ${ }^{18,40}$ An antibody to MMP-9 has been shown to reduce the size of an infarct in a model of stroke in rats. ${ }^{27}$ Genetically modified animals lacking the MMP-9 gene have less damage to the BBB and smaller strokes than wild-type animals. ${ }^{3}$ These studies have been focused on the acute phase of stroke, and the long-term effects of these agents are unclear. We have used MR imaging to document early injury to the BBB noninvasively and to measure the size of the infarct at 48 hours. After the initial MR imaging studies, animals were tested behaviorally for 4 weeks. We found that treatment for 3 days with the broad-spectrum inhibitor BB-1101 dramatically reduced the initial BBB opening, had no effect on infarct size, and caused a slowing in recovery (Sood et al., unpublished data). The use of these agents for the early prevention of $\mathrm{BBB}$ opening must include long-term follow-up to identify a safe therapeutic window for their use. In addition, as more is learned about the MMPs involved in the recovery phase, selective MMP inhibitors can be developed and tested.

\section{Future Studies}

Many laboratories are now engaged in studies on the role of MMPs in damage to the BBB that results in vasogenic edema. Rational use of MMP inhibitors to protect from MMP-mediated proteolysis of TJPs in stroke treatment will require a better understanding of the mechanisms of the proteolysis of TJPs by MMPs. Synthetic inhibitors have shown promise in controlling damage to both the extracellular matrix proteins that comprise the basal lamina and the tight junctions. Which inhibitors will be useful clinically remains an open question. Newer ones are being developed that act selectively against one or more of the MMPs, sparing others; such inhibitors are important because the beneficial effects may be due to one 


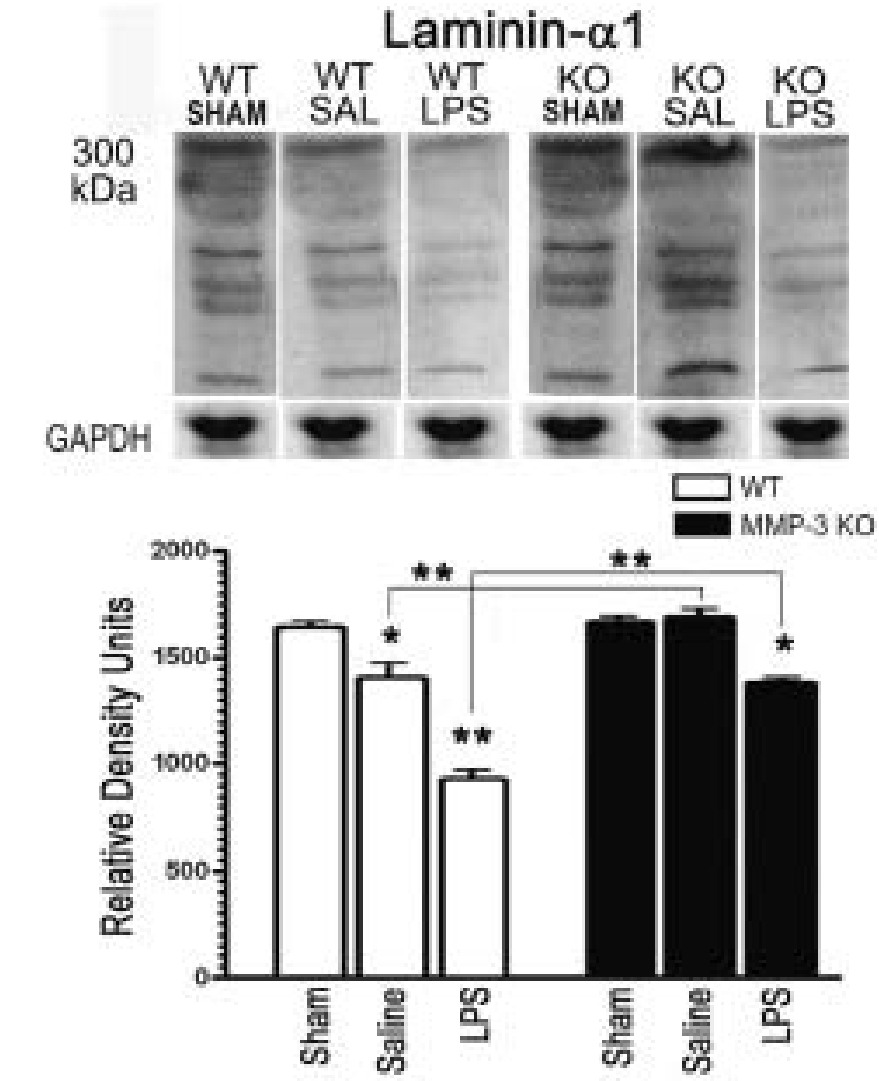

FIG. 6. Western gel blot and bar graph demonstrating the effect of LPS on the basal lamina protein laminin- $\alpha 1$.

Representative bands from one mouse are shown in the upper portion of the figure. The major form of $300 \mathrm{kD}$ laminin- $\alpha 1$ was measured by densitometry. Densitometric analysis 24 hours after LPS or saline injection showed that LPS injection resulted in a significant decrease in laminin- $\alpha 1$ levels in both wild-type (WT) and MMP-3 knockout (KO) mice (three animals for each WT and KO group, $* p<0.05$ and $* * p<0.001)$. However, laminin- $\alpha 1$ levels decreased to a lesser extent in the MMP-3 KO than in the WT mice. The lower bands are likely degradation products. (From Gurney et al., 2006.)

group of enzymes, whereas others may cause the damaging effects. Studies are needed to define the timing of MMP inhibitor treatment so that the beneficial effects of the MMPs are not blocked. Research may require the short-term use of these agents in the early stages of stroke or reperfusion. With newer methods of developing and screening potential therapies and advances in the use of MR imaging to characterize tissue damage, it may be possible to reduce damage to the extracellular matrix and TJPs, which in turn would reduce the risk of vasogenic edema and hemorrhage in cerebral ischemia and expand the therapeutic window for the use of thrombolysis in stroke.

\section{References}

1. Abbott NJ, Ronnback L, Hansson E: Astrocyte-endothelial interactions at the blood-brain barrier. Nat Rev Neurosci 7: 41-53, 2006

2. Anonymous: Tissue plasminogen activator for acute ischemic stroke. The National Institute of Neurological Disorders and Stroke rt-PA Stroke Study Group. N Engl J Med 333: 1581-1587, 1995

3. Asahi M, Asahi K, Jung JC, del Zoppo GJ, Fini ME, Lo EH: Role for matrix metalloproteinase 9 after focal cerebral ischemia: effects of gene knockout and enzyme inhibition with BB-94. J Cereb Blood Flow Metab 20:1681-1689, 2000

4. Belayev L, Busto R, Zhao W, Ginsberg MD: Quantitative evaluation of blood-brain barrier permeability following middle cerebral artery occlusion in rats. Brain Res 739:88-96, 1996

5. Brown PD: Synthetic inhibitors of matrix metalloproteinases, in Parks WC, Mecham RP (eds): Matrix Metalloproteinases. London: Academic Press, 1998, pp 243-261

6. Cao J, Rehemtulla A, Bahou W, Zucker S: Membrane type matrix metalloproteinase 1 activates pro-gelatinase A without furin cleavage of the N-terminal domain. J Biol Chem 271: 30174-30180, 1996

7. Cunningham LA, Wetzel M, Rosenberg GA: Multiple roles for MMPs and TIMPs in cerebral ischemia. Glia 50:329-339, 2005

8. del Zoppo GJ, Copeland BR, Anderchek K, Hacke W, Koziol JA: Hemorrhagic transformation following tissue plasminogen activator in experimental cerebral infarction. Stroke 21: 596-601, 1990

9. Fisher M, Adams RD: Observations on brain embolism with special reference to the mechanism of hemorrhagic infarction. J Exper Neurol Neuropath 10:92-96, 1951

10. Furuse M, Fujita K, Hiiragi T, Fujimoto K, Tsukita S: Claudin1 and -2 : novel integral membrane proteins localizing at tight junctions with no sequence similarity to occludin. J Cell Biol 141:1539-1550, 1998

11. Gearing AJ, Beckett P, Christodoulou M, Churchill M, Clements J, Davidson AH, et al: Processing of tumor necrosis factor-alpha precursor by metalloproteinases. Nature 370: 555-557, 1994

12. Gurney KJ, Estrada EY, Rosenberg GA: Blood-brain barrier disruption by stromelysin-1 facilitates neutrophil infiltration in neuroinflammation. Neurobiol Dis 23:87-96, 2006

13. Hatashita S, Hoff JT: Brain edema and cerebrovascular permeability during cerebral ischemia in rats. Stroke 21:582-588, 1990

14. Hirase T, Staddon JM, Saitou M, Ando-Akatsuka Y, Itoh M, Furuse M, et al: Occludin as a possible determinant of tight junction permeability in endothelial cells. J Cell Sci 110: 1603-1613, 1997

15. Hornig CR, Bauer T, Simon C, Trittmacher S, Dorndorf W: Hemorrhagic transformation in cardioembolic cerebral infarction. Stroke 24:465-468, 1993

16. Kuroiwa T, Ting P, Martinez H, Klatzo I: The biphasic opening of the blood-brain barrier to proteins following temporary middle cerebral artery occlusion. Acta Neuropathol 68:122-129, 1985

17. Lapchak PA, Chapman DF, Zivin JA: Metalloproteinase inhibition reduces thrombolytic (tissue plasminogen activator)-induced hemorrhage after thromboembolic stroke. Stroke 31: 3034-3040, 2000

18. Lee SR, Kim HY, Rogowska J, Zhao BQ, Bhide P, Parent JM, et al: Involvement of matrix metalloproteinase in neuroblast cell migration from the subventricular zone after stroke. J Neurosci 26:3491-3495, 2006

19. Lo EH, Broderick JP, Moskowitz MA: tPA and proteolysis in the neurovascular unit. Stroke 35:354-356, 2004

20. Mudgett JS, Hutchinson NI, Chartrain NA, Forsyth AJ, McDonnell J, Singer II, et al: Susceptibility of stromelysin 1-deficient mice to collagen-induced arthritis and cartilage destruction. Arthritis Rheum 41:110-121, 1998

21. Nagase H: Activation mechanisms of matrix metalloproteinases. Biol Chem 378:151-160, 1997

22. Nelson AR, Fingleton B, Rothenberg ML, Matrisian LM: Ma- 


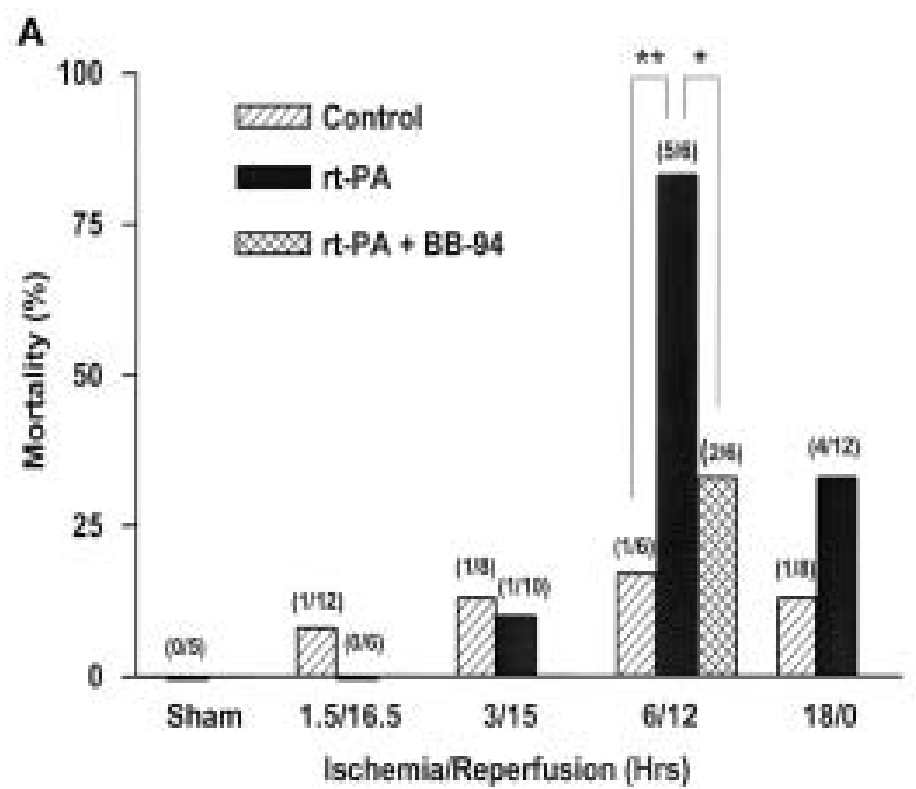

B

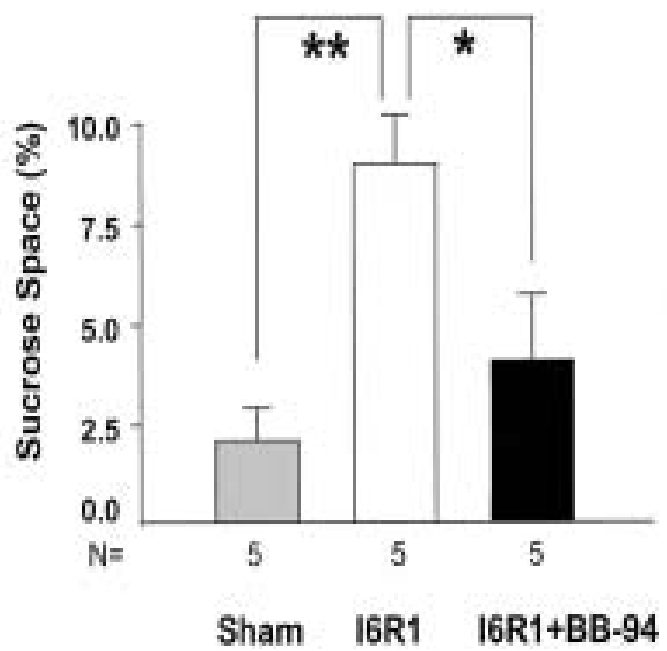

FIG. 7. Bar graphs showing effects of the broad-spectrum MMP inhibitor BB-94 on the BBB in rats with cerebral ischemia induced using the suture model. Treatment with rt-PA resulted in increased deaths, which were blocked by BB-94. A: Effect of various intervals of ischemia and reperfusion with and without rt-PA treatment on different outcome parameters. In delayed reperfusion, that is, after 6 hours of ischemia, the number of deaths increased markedly in rt-PA- treated animals. Animals that had undergone permanent occlusion had much lower rates of death, which was only minimally affected by rt-PA. Groups were defined according to different ischemic intervals of 0 (sham), 1.5, 3, and 6 hours. A group of animals with permanent occlusion was included. Bars represent the means \pm standard errors of the means. Significant differences between treated and untreated animals are indicated by an asterisk. The influence of different ischemic intervals on outcome parameters was analyzed using analysis of variance. B: Opening of the BBB as measured by sucrose space in rats that had undergone 6 hours of ischemia and 1 hour of reperfusion (I6R1).

Compared with sham-operated animals, BBB permeability was markedly increased in untreated rats $(* * p<0.01)$.

Note that BB-94 administered 2 and 5 hours after MCAO markedly decreased BBB opening in the sham $(* * \mathrm{p}<0.01)$ and in the treated rats $(* \mathrm{p}<0.05)$. Gray bars represent saline-treated animals; black bars, rt-PA-treated animals. $\mathrm{N}=$ number of rats. (Figure modified from Pfefferkorn et al., 2003.)

trix metalloproteinases: biologic activity and clinical implications. J Clin Oncol 18:1135-1149, 2000

23. Ning M, Furie KL, Koroshetz WJ, Lee H, Barron M, Lederer $\mathrm{M}$, et al: Association between tPA therapy and raised early matrix metalloproteinase-9 in acute stroke. Neurology 66: 1550-1555, 2006

24. Opdenakker G, Van den Steen PE, Dubois B, Nelissen I, Van Coillie E, Masure S, et al: Gelatinase B functions as regulator and effector in leukocyte biology. J Leukoc Biol 69:851-859, 2001

25. Pfefferkorn T, Rosenberg GA: Closure of the blood-brain barrier by matrix metalloproteinase inhibition reduces rtPA-mediated mortality in cerebral ischemia with delayed reperfusion. Stroke 34:2025-2030, 2003

26. Robert AM, Godeau G: Action of proteolytic and glycolytic enzymes on the permeability of the blood-brain barrier. Biomedicine 21:36-39, 1974

27. Romanic AM, White RF, Arleth AJ, Ohlstein EH, Barone FC: Matrix metalloproteinase expression increases after cerebral focal ischemia in rats: inhibition of matrix metalloproteinase-9 reduces infarct size. Stroke 29:1020-1030, 1998

28. Rosenberg GA: Matrix metalloproteinases in neuroinflammation. Glia 39:279-291, 2002

29. Rosenberg GA, Estrada EY, Dencoff JE: Matrix metalloproteinases and TIMPs are associated with blood-brain barrier opening after reperfusion in rat brain. Stroke 29:2189-2195, 1998
30. Rosenberg GA, Kornfeld M, Estrada E, Kelley RO, Liotta LA, Stetler-Stevenson WG: TIMP-2 reduces proteolytic opening of blood-brain barrier by type IV collagenase. Brain Res 576: 203-207, 1992

31. Rosenberg GA, Mun-Bryce S, Wesley M, Kornfeld M: Collagenase-induced intracerebral hemorrhage in rats. Stroke 21: 801-807, 1990

32. Rosenberg GA, Navratil M, Barone F, Feuerstein G: Proteolytic cascade enzymes increase in focal cerebral ischemia in rat. J Cereb Blood Flow Metab 16:360-366, 1996

33. Sato H, Takino T, Okada Y, Cao J, Shinagawa A, Yamamoto E, et al: A matrix metalloproteinase expressed on the surface of invasive tumor cells. Nature 370:61-65, 1994

34. Strongin AY, Collier I, Bannikov G, Marmer BL, Grant GA, Goldberg GI: Mechanism of cell surface activation of 72-kDa type IV collagenase. Isolation of the activated form of the membrane metalloprotease. J Biol Chem 270:5331-5338, 1995

35. Wang YF, Tsirka SE, Strickland S, Stieg PE, Soriano SG, Lipton SA: Tissue plasminogen activator (tPA) increases neuronal damage after focal cerebral ischemia in wild-type and tPA-deficient mice. Nat Med 4:228-231, 1998

36. Wolpert SM, Bruckmann H, Greenlee R, Wechsler L, Pessin MS, del Zoppo GJ: Neuroradiologic evaluation of patients with acute stroke treated with recombinant tissue plasminogen activator. The rt-PA Acute Stroke Study Group. AJNR Am J Neuroradiol 14:3-13, 1993

37. Yang GY, Betz AL: Reperfusion-induced injury to the blood- 
brain barrier after middle cerebral artery occlusion in rats. Stroke 25:1658-1665, 1994

38. Yang Y, Estrada EY, Thompson JF, Liu W, Rosenberg GA: Matrix metalloproteinase-mediated disruption of tight junction proteins in cerebral vessels is reversed by synthetic matrix metalloproteinase inhibitor in focal ischemia in rat. J Cereb Blood Flow Metab 27:697-709, 2007

39. Yong VW: Metalloproteinases: mediators of pathology and regeneration in the CNS. Nat Rev Neurosci 6:931-944, 2005

40. Zhao BQ, Wang S, Kim HY, Storrie H, Rosen BR, Mooney DJ, et al: Role of matrix metalloproteinases in delayed cortical responses after stroke. Nat Med 12:441-445, 2006

Manuscript submitted January 15, 2007.

Accepted March 23, 2007.

Address reprint requests to: Gary A. Rosenberg, M.D., Department of Neurology, MSC10 5620, 1 University of New Mexico, Albuquerque, New Mexico 87131-0001. email: Grosenberg@ salud.unm.edu. 